RETRACTION

doi:10.1038/nature18613

\title{
Retraction: Odour receptors and neurons for DEET and new insect repellents
}

Pinky Kain, Sean Michael Boyle, Sana Khalid Tharadra, Tom Guda, Christine Pham, Anupama Dahanukar \&

Anandasankar Ray

Nature 502, 507-512 (2013); doi:10.1038/nature12594

We are retracting this Article because we no longer have confidence in data that support one of our key conclusions. In this Article we reported four advances in insect repellency: identification of olfactory neurons in Drosophila melanogaster that participate in repellency to $\mathrm{N}, \mathrm{N}$-diethyl-meta-toluamide (DEET); identification of an ionotropic receptor, Ir40a, expressed in these neurons required for avoidance to DEET; development of a chemical informatics method of identifying shared structural features from known behavioural repellents; and validation of a series of computationally identified natural chemicals as repellents for flies and mosquitoes. We no longer have confidence in data supporting that Ir40a is a DEET receptor. Upon reanalysis, the original calcium imaging (GCAMP) data show movement artefacts and background effects that we originally missed, which seriously undermine our confidence in Ir40a responses to DEET. In addition, Supplementary Fig. $5 \mathrm{~b}$ presents several inappropriately re-used panels.

Upon learning that A. F. Silbering et al. ${ }^{1}$ did not find defects in DEET aversion in Ir40a mutant flies, we repeated many of the original behaviour experiments. Although we confirmed significant behavioural differences in Ir40a cell-silenced flies (Ir40a-Gal4;UAS-TNTG), as reported in Fig. 2d, we have been unable to replicate observations of behavioural experiments using Ir40a-Gal4;UAS-RNAi flies. Therefore, with the exception of author Pinky Kain, we no longer have confidence in the conclusions of Figs 2, 3 and 5c, and Supplementary Fig. 5. We remain confident of the chemical informatics analyses and the identification of new repellents, which have been successfully repeated in our laboratory and by others, as reported in Figs 4, 5d and e, 6, and Supplementary Figs 2 and 6-9. Although it may still be possible that Ir40a does respond to DEET, given the issues listed above, all authors except Pinky Kain wish to retract this Article in its entirety. We deeply regret these circumstances and apologize to the scientific community.

1. Silbering, A. F. et al. Ir40a neurons are not DEET detectors. Nature 534, E5-E7, http://dx.doi.org/10.1038/nature18321 (2016). 